
\title{
CS Research Suare \\ Prediction of COVID-19 Prognosis By Heterogeneity Analysis Based on Chest CT Scans
}

\section{Bo Yang}

General Hospital of Central Theater Command

\section{Bei Zhang}

Shaanxi Provincial Tumor Hospital

\section{Lichen Gao}

General Hospital of Central Theater Command

Jian Zhang

General Hospital of Central Theater Command

Huaiming Qiu

General Hospital of Central Theater Command

Wencai Huang ( $\nabla$ dr_hwang@163.com )

General Hospital of Central Theater Command

\section{Research}

Keywords: COVID-19, heterogeneity, histogram analysis, nomogram, clinical outcome

Posted Date: January 19th, 2021

DOl: https://doi.org/10.21203/rs.3.rs-147571/v1

License: @ (i) This work is licensed under a Creative Commons Attribution 4.0 International License. Read Full License 


\section{Abstract}

\section{Background}

Ground-glass opacity (GGO) and consolidation opacity (CLO) are the common CT lung opacities, and their heterogeneity may have potential for prognosis in COVID-19 patients. This study aimed to estimate clinical outcome in individual COVID-19 patient by using histogram heterogeneity analysis based on CT opacities.

\section{Methods}

71 COVID-19 cases' medical records were retrospectively reviewed from a designated hospital in Wuhan, China, from January 24th to February 28th at the early stage of pandemic. Two characteristic lung abnormity opacities, GGO and CLO were drawn on CT images to identify the heterogeneity by using quantitative histogram analysis. The parameters (mean, mode, kurtosis, skewness) were derived from histograms to evaluate the accuracy of clinical classification and outcome prediction. Nomograms were built to predict the risk of death and median length of hospital stays (LOS), respectively.

\section{Results}

A total of 57 cases were eligible for the study cohort after exclusion 14 cases. The most highly frequency of lung abnormalities was GGO mixed with CLO in both survival population (26 in 42, 61.9\%) and died population (10 in $15,66.7 \%)$. The best performance heterogeneity parameters to discriminate severe type from mild/moderate counterparts were as following: GGO_skewness: specificity $=66.67 \%$, sensitivity $=78.12 \%$, AUC=0.706; CLO_mean: specificity $=70.00 \%$, sensitivity $=76.92 \%, A U C=0.746$. Nomogram based on histogram parameters has the ability to predict the individual risk of death and the prolonged median LOS of COVID-19 patients. C-indexes were 0.763 and 0.888 for risk of death and prolonged median LOS, respectively.

\section{Conclusions}

The histogram analysis method based on GGO and CLO has the ability for individual risk prediction in COVID-19 patients.

\section{Introduction}

The 2019 novel coronavirus disease (COVID-19) is a lethal disease with much infectivity caused by severe acute respiratory syndrome coronavirus 2 (SARS-CoV-2)(1). The COVID-19 pandemic has become a global public health emergency infecting exceeding 30,000,000 cases and resulting in more than 900,000 deaths of the disease (update to Sept 19nd, 2020), according to data from the Center for Systems Science and Engineering at Johns Hopkins University.(2).

Chest CT has the advantages of timely, fast and highly sensitivity, which plays an important role in screening of suspected infected patients and monitoring the lung changes of confirmed patients. Previous studies have reported that COVID-19 patients have similar appearance in CT imaging, the most common CT features are (1) single or multiple round or oval shape pure ground-glass opacities (GGO) in subpleural area or peribronchial areas or both, (2) single or diffuse consolidation opacities (CLO) in above-mentioned areas, (3) mixed GGO and CLO patchy or diffuse distribution in lateral or bilateral pulmonary lobes. Intra or interlobular septal thickening or 
dilated bronchi were frequently seen in these patients. The semi-quantitative score based on CT imaging has been wildly used to evaluate the severity of pulmonary infection, and this score system has been confirmed consistent with clinical conditions. However, the relatively low accuracy and specificity of CT semi-quantitative score are the main shortages for onset typing diagnosis or predicting outcome during treatment course (3). Thus, it is necessary to develop a quantitative analysis approach to better provide more valuable information for treatment decision making.

Histogram analysis is a quantitative method, which is widely employed in the grading and prognostic diagnosis of tumors, inflammations and other diseases. Histogram parameters, such as skewness, kurtosis, mode, and mean, can provide more voxel distribution information, reflecting subtle histological heterogeneity. In the COVID19 patients, the severity of the clinical condition is mainly related to different pathological manifestations (such as inflammatory exudation or edema or fibrosis). These pathological differences can be reflected on CT images (such as GGO or CLO), which can be identified by radiologists or other clinicians due to different CT densities. However, the subtle histological heterogeneity inside either GGO or CLO on chest CT imaging is still unknown. The method of opacities histogram analysis, which is based on voxel distribution, which is more accurate and reliable in the reflection of the heterogeneity of opacities zone, and may provide more quantitative information for COVID19 diagnosis and prognosis.

In this study, we reviewed the data of 71 COVID-19 patients from a designated hospital in Wuhan, China. We aimed to develop a quantitative analysis approach to evaluate the severity and outcome of COVID-19. We firstly investigated whether histogram analysis of CT images base on GGO and/or CLO heterogeneity could accurately predict clinical classification and outcome in COVID-19. Furthermore, we try to build a comprehensive and effective nomogram based on histogram parameters to predict the individual risk of death and the prolonged median length of hospital stays (LOS) in COVID-19 patients.

\section{Methods}

\section{Clinical data collection}

In this retrospective study,the clinical and chest CT imaging findings 71 patients in total were reviewed for from a designated hospital in Wuhan, China, from January 24th to February 28th at the early stage of pandemic. All patients were confirmed diagnosed with COVID-19 by RT-PCR. The onset clinical classification and the following treatment of each patient were strictly carried out by clinicians according to Diagnosis and Treatment Protocols of COVID-19 Infection (8th edition) (hereafter referred to as Protocols-Ver8) published by National Health Commission of the People's Republic of China. All the patients have clear course with a definite outcome (survival or die) written in the medical records. In order to ensure data accuracy, a follow-up survey was performed at 30 days post the survival patients discharged from hospital.

This study was approved by the Ethics Committee of the General Hospital of Central Theater Command and was conducted according to Health Insurance Portability and Accountability Act regulations. In addition, written consent was waived by the Ethics Committee of the General Hospital of Central Theater Command.

The retrospective study design and workflow diagram with patient inclusion and exclusion were shown on Figure 1. Clinical medical record, laboratory testing, chest CT imaging were collected from PACS using a standardized data collection. All data were reviewed by two investigators independently to verify data accuracy. 
Chest CT scan was performed on 64-slice multiple spiral CT (Insitum 64S, SinoVision Company, Beijing, China) without contrast. Briefly, Chest CT images were acquired during inspiratory breath-hold. CT parameters were as follows: tube voltage $120 \mathrm{kV}$, automatic tube current, pitch of 1.3, matrix $512 \times 512$, slice thickness 0.625 $\mathrm{mm}$, spacing $0.625 \mathrm{~mm}$, and All original images were reconstructed to $1.25 \mathrm{~mm}$ section on workstation and reviewed on PACS.

Image reviewed and semi-quantitative score

Two radiologists who were blinded to clinical data independently reviewed the chest CT images with axial section to evaluate imaging features, including the density heterogeneity (GGO or CLO), lung distribution and border definition. Intra or interlobular septal thickening or subpleural linear opacity was also reviewed. A semiquantitative score system was performed visually to evaluate the degree of involvement of each lung lobe based on the percentage of abnormality opacities. Each lobe scored in a four-point scale: score 1, 1-25\% involvement; score 2, 26-50\% involvement; score 3; 51-75\% involvement; score 4, 76-100\% involvement. Total lung scores were calculated as the sum of each lobe score.)(4). For cases in which the two radiologists' findings were discrepant, a consensus was allocated.

\section{Quantitative Histogram Analysis}

Two observers who were blinded to clinical data performed quantitative imaging analysis independently. As described in previous studies(5), regions of interest (ROI) for the ground-glass opacity (GGO), consolidation opacity (CLO) were respectively drawn in each image section using MRIcroN software (https://www.nitrc.org/projects/mricron). For ROI boundaries definition were as following: i) GGO were defined with reference to the high-density intensity areas consisting of a hazy opacity that does not obscure the underlying bronchial structures or pulmonary vessels; ii) CLO were defined a higher density than GGO consisting of consolidation and infiltrates pervade the lungs, leaving few recognized air spaces; iii) Intra or interlobular septal thickening or subpleural linear opacity was classified as GGO or CLO; iv)ROI were carefully drawn to avoid areas of macro-vessels or trachea/dilated bronchus or pleural effusion; v) Voxels in each ROI value was normalized by the mean of ROI of the thoracic artery. For cases in which the two observers' findings were discrepant, a consensus was allocated.

The histogram analysis was performed by using the MATLAB (v9.4.0; MathWorks). ROI histograms were plotted with relative CT value on the $x$-axis, with a bin size of 15 , and the $y$-axis expressed as a percentage of each bin in the total ROI CT value.

The following parameters were derived from histograms: (a) mean; (b) mode, which equals the peak height position; (c) kurtosis, which is the degree of peakedness of a distribution; and (d) skewness, which is a measure of the degree of asymmetry of a distribution.

\section{Statistical analysis and nomogram}

Receiver operating characteristic (ROC), Cox proportional hazards regression models was conducted in SPSS for Windows (Version 24.0, Chicago, IL, USA). The nomogram was built by using R software (Version 3.5.0, https://www.r-project.org/). Continuous variables were expressed as mean \pm standard deviation (SD). 
The optimal diagnostic cut-offs were derived from the ROC analysis with the area under the curve (AUC) in order to distinguish the diagnostic sensitivity and specificity of the mild/moderate type and severe type in COVID-19 patents, as well as to distinguish survival with dead population.

For survival prediction, multivariable time-to-death event analysis was performed using Cox proportional hazards regression models to obtain odds ratio (OR) and develop a nomogram using weighted estimators corresponding to each covariate derived from Cox regression coefficients and estimates of variance constructed using $R$ software with the rms package as described previously(6). For median LOS prediction, the prolonged LOS (>30 days) was defined as the end-event.

Validation of the nomogram was assessed by discrimination and calibration. Harrell's C statistic was calculated by 1,000-fold bootstrap resampling iterations to an initial fitted Cox model in the derivation set. The maximum Cindex value is 1.0 , which indicates a perfect prediction model whereas 0.5 indicates a random chance to correctly predict outcome by the model. Bootstraps with 1,000 resamples were used for validation to correct the $\mathrm{C}$-index and explain the variance due to over-optimism. For individual patients, the sum of nomogram scores for each variable will transform to survival probability.

\section{Results}

\section{Clinical cases data}

Table 1 describes the baseline demographic and initial symptoms of the investigated population.

An aggregate of 71 cases confirmed with COVID-19 were retrospectively studied. 14 cases were excluded due to poor image quality or without clear CT lung abnormalities. 57 cases (38 men and 19 women, average age, 67.00 years \pm 18.89 , range from 31-97 years) were enter into the final analysis. According to the Protocols-Ver8, 36 cases (63.2\%) were classified as severe type, while 21 cases (36.8\%) as mild and moderate type. 15 cases (26.3\%) died during hospitalization and 42 cases (73.7\%) were discharged from hospital. All the discharged cases were survival at 30 days follow-up survey. The mean LOS for survival population was $35.29 \pm 18.04$ days, while the mean overall survival (OS) of died population was $31.67 \pm 11.87$ days.

The patients in this study population were present at least one respiratory symptoms symptom, and/or more symptoms onset of illness. The most common initial symptoms were fever (48 cases, $84.2 \%$ ), cough (33 cases, $57.9 \%$ ) the other initial symptoms include hypodynamia, chest tightness, muscle pain, diarrhea, headache, etc. Noting that, 5 in 7 cases present with diarrhea were died in this study population.

\section{Chest CT findings and Semi-quantitative CT score}

The frequencies of major chest CT findings, GGO and/or CLO, were analyzed in whole study population, each group (survival vs. died, severe vs. mild/moderate) of population independently, summarized in Table 2. Overall, 13 pure GGO, 8 pure CLO and 36 GGO mixed with CLO were present in the whole study population. The most highly frequency of lung abnormality was GGO mixed with CLO in both survival population (26 in 42, 61.9\%) and died population (10 in 15,66.7\%). With regard to the predominance of the lung opacity, survival population were more likely to show more cases of GGO predominance over CLO (GGO vs. CLO, 61.9\% vs 38.0\%), while died population showed almost equal cases between GGO and CLO predominance. In survival population, the highly frequency infected lobes were at the right and left lower lobe (92.9\% and 83.3\%). In died population, both right 
and left lower lobes were infected in all 15 cases $(100 \%)$. The CT severity score was considerably higher in died cases than survival cases [died vs survival cases: $7.41 \pm 4.18$ vs $4.26 \pm 2.68$ ]. Only died population shown the score 4 [right upper lobe: $3(20 \%)$; right lower lobe: 3(20\%); left upper lobe: $2(13.3 \%)$; left lower lobe: $4(26.7 \%)$ ]. The frequencies and CT semi-quantitative score were also analyzed in severe, mild/moderate populations (Table 2).

\section{Semi-quantitative CT score for clinical classification}

ROC curves were presented in Fig. 2. The CT severity score has the ability to discriminate the clinical classification and outcome. The sensitivity and specificity for diagnosis severe type from mild/moderate counterparts were $88.24 \%$ and $56.52 \%$, respectively (AUC, 0.753 ). For outcome, the sensitivity and specificity were $86.67 \%$ and $52.38 \%$, respectively (AUC, 0.729 ).

\section{Histogram quantitatively analysis for clinical classification}

ROC curves are presented in Fig. 3 and details of each histogram parameter are presented in Table S1

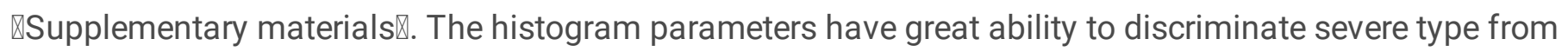
mild/moderate type COVID-19. The significant diagnostic value of histogram parameters derived from ROC analysis was as following: GGO_skewness (specificity $=66.67 \%$, sensitivity $=78.12 \%, A U C=0.706, p=0.0094$ ), GGO_mode(specificity $=77.78 \%$, sensitivity $=59.38 \%$, AUC $=0.707, p=0.0059$ ), GGO_mean (specificity $=83.33 \%$, sensitivity $=62.5 \%, A U C=0.742, p=0.0008$ ), CLO_mode (specificity $=80.00 \%$, sensitivity $=57.69 \%$, AUC $=0.680$, $p=0.0024$ ), and CLO_mean (specificity $=70.00 \%$, sensitivity $=76.92 \%$, AUC $=0.746, p=0.001$ ).

COX proportional hazards mode evaluates for individual risk of death prediction in COVID-19 and nomogram

The Cox proportional hazard analysis with ten variables (8 histogram parameters with age and gender) is presented in Table S2 $\varangle$ Supplementary materials $₫$. Significant results were obtained for GGO_kurtosis (OR=7.607 $p=0.002)$, GGO_skewness ( $\mathrm{OR}=0.122, p=0.026), \mathrm{GGO}$-mode( $\mathrm{OR}=3.566, p=0.007), \mathrm{GGO}$-mean $(\mathrm{OR}=0.254$, $p=0.005)$, $C L \_m o d e(O R=0.609, p=0.036)$, and $C L \_m e a n(O R=2.016, p=0.007)$. The result of nomogram on the basis of Cox proportional hazard analysis was shown in Figure.4. Internal validation using the bootstrap method showed that the $\mathrm{C}$-index for the model was 0.763 . The calibration curve is shown in Fig. S1. It reveals no deviations from the reference line and no need for recalibration. We have shown two examples of severe type pneumonia from died and survival cohort respectively (Fig.5). A 60 years old female patient of died cohort with a total of histogram parameters $\approx 137$ points, which corresponds to a 30 -day risk of death probability of $79 \%$, while a 57 year old male patient of survival cohort with a total of histogram parameters $\approx 126$ points, which corresponds to a 30 -day risk of death probability of $35 \%$.

COX proportional hazards mode evaluates for individual prolonged median LOS prediction in COVID-19 and nomogram

The Cox proportional hazard analysis with ten variables (8 histogram parameters with age and gender) is

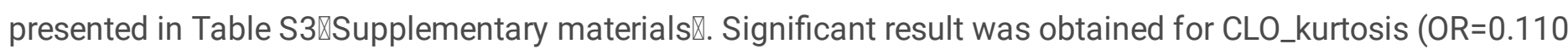
$p=0.017)$. Trend result was obtained for GGO_mode ( $\mathrm{OR}=0.290 p=0.071)$. The result of nomogram on the basis of Cox proportional hazard analysis was shown in Fig.6. Internal validation using the bootstrap method showed that the $\mathrm{C}$-index for the model was 0.888 . The calibration curve is shown in Fig. S2. It reveals no deviations from the reference line and no need for recalibration. 


\section{Discussion}

In the present study, we investigated whether histogram analysis based on chest CT imaging could provide a quantitative approach to predict clinical classification and outcome of patients confirmed with COVID-19. Our results showed that the histogram parameters have the ability to quantify the heterogeneity of GGO and CLO, and have better diagnostic performance in typing diagnosis and predicting outcome. Furthermore, we have built a nomogram based on Cox proportional hazard analysis with ten variables (8 histogram parameters with age and gender) to predict the 30 days survival probability of COVID-19 patients, and median LOS for survival subgroup.

Chest CT is considered as an essential and powerful tool during the course of diagnose and treatment in COVID19 , for the CT scan can find small characteristic patchy shadows or interstitial changes at the early of the disease, as well as monitor the pneumonia changes during the course of treatment. Even more, CT precedes positive reverse transcriptase polymerase chain reaction testing (RT-PCR) in some cases $(3,4)$. Many studies reported the COVID-19 have similar appearance on CT imaging, and performed semi-quantitative score system based on the infectious involvement of lung lobes. Of chest CT for COVID-19, the positive predictive value (PPV) is estimated at $92 \%$ and negative predictive value (NPV) is estimated at $42 \%$, which is consistent with the high pretest probability for the disease (i.e., $85 \%$ prevalence by RT-PCR) (3). In another study, PPV is only $46 \%$, but the NPV is $99 \%$ for COVID-19 diagnosis by using chest CT(7). Inui S et al reported that there were significant difference of CT severe score between symptomatic and asymptotic cases in an environmentally homogeneous cohort from the cruise ship "Diamond Princess"(8). In our study, we found that the CT severe score has a high sensitivity (88.24\%) but a low specificity (56.52\%) to discriminate pneumonia type (severe vs mild/moderate). The relatively low specificity of CT findings for COVID-19 pneumonia diagnosis will reinforcing the need to develop a quantitively approach with overall considering the CT imaging density information.

GGO and CLO are the typical lung abnormalities on CT imaging of COVID-19, and are highly heterogeneity at histology and cellular level. Ground-glass opacity (GGO) refers to hazy areas with mildly increased density in lungs, but without obscuration of bronchial and vascular margins, which generally is due to destruction of alveolar walls and filling of the cavity with serous, cells and hemorrhage as well as local interstitial fibrosis. (9). Consolidation opacity is caused by any pathologic process that diffuse alveolar injury in both lungs with fibrous mucinous exudate. Then lung Alveolar epithelial cells fall off and a transparent membrane is formed. In severe or critical COVID-19 cases, SARS-CoV-2 invades the pulmonary capillary endothelial cells and causes the epithelialendothelial (air-blood) barrier to break. A large number of lymphocytes and plasma exudation are seen in the alveolar cavity, which causes acute respiratory distress syndrome.(10). In the autopsy studies of COVID-19, there reported that pathological feature of lung is diffuse alveolar damage with prominent fibrous mucus exudate and mucous plug formation $(11,12)$. The heterogeneous pathological pattern of lung will make the opacities extremely inhomogeneous density at CT images. Timely quantification of the heterogeneity of the two typical opacities will be a potential approach for initial prognostic prediction and risk stratification for treatment decisions in COVID-19 pneumonia patients. Histogram analysis is a concise and accuracy method for quantification of lung CT imaging(13). The method of opacities histogram analysis based on voxel distribution is more accurate and reliable in the reflection of the heterogeneity of opacities zone. The method is able to avoid the sampling error resulted from $\mathrm{ROI}$ in local area as much as possible, and provide more quantitative information for COVID-19 pneumonia diagnosis and prognosis. In our study, severe type prediction in COVID-19 pneumonia is great performance by using the indexes value derived from histogram analysis was as following: CLO_mean (NPV=70.00\%, PPV=89.8\%), with a 25.6\% prevalence of severe type reported by Fu LW et al (14). 
Many studies were done to subtract independent factor for diagnosis and prognosis prediction in COVID-19 pneumonia patients. Mao R reported that digestive symptoms are the significant hazard factor for severe COVID19 pneumonia patients (15). The relationship between clinical outcome with CT features is still unclear. In our study population, we found that severe pneumonia patients with same CT severe score may have different outcome. We done the Cox proportional hazards model with combine important factors and then built prognostic nomograms to quantify risk as precisely as possible in order to individually predict outcomes of COVID-19 pneumonia patents.

Nomograms are commonly used tools to estimate prognosis in medicine, which are capable of generating $\backslash$ an individual numerical probability of a clinical event with integration of diverse prognostic and determinant variables. Many factors will challenge nomogram utility, is list as following: i), nomograms assume that outcomes do not change over time; ii) heavily censor data; iii) secular trends with capture of more events, and iv) conveying results to clinical-decision making for an individual patient (16). We enrolled adult inpatients from designated hospitals for COVID-19, which has the same treatment protocol and has a clear outcome (dead or discharged) at the early phase of the outbreak. Our study cohort is a better model for nomogram utility for the simple end events, standard treatment and 30-days follow-up survey post discharged. We identify the GGO and CLO heterogeneity parameters have different impaction for individual clinical outcome prediction. GGO_mode is a major weighted factor both in the risk of death and prolonged median LOS. CLO_kutosis is a minor weighted factor in prediction of risk of death but major weighted in LOS prediction. We speculate GGO and CLO parameters have relationship with pathology findings in different cohort population. More radiology-pathology control studies need to be done to find out the underline mechanisms.

The influence of gender on morbidity and mortality of COVID-19 is a critical topic. Many studies indicated that male has a high ratio in COVID patients(17-18). In a recent study, male are more vulnerable with worse outcomes and death, irrespective of age, with COVID-19(19). In our study, we found that although the gender is not an independent risk factor for COVID-19 patenta, male patients has high risk $(O R=10,716)$ compared to female patients with same pulmonary infection degree. It has been accepted that varied behavior, hormone responses, and likelihood to be infected by disease may lead to distinct profiles of pneumonia-associated morbidity for male and female(20), and this will also applicable for the COVID-19 pandemic.

There are some limitations in this study. One of the main limitations, we did not conduct the longitudinal study but only analysis the onset of COVID-19 pneumonia. The frequency of imaging findings also depends on the time of imaging of the infected patinets(21). Second, we did not include asymptotic patients in this retrospective study. In addition, for the relatively small sample size, the complications (e.g., hypertension, diabetes, coronary artery disease) which had been proved the risk factor of morbidity of COVID-19(22) were not using for the cox hazard model.

\section{Conclusions}

We conclude that: i) although initial chest CT with semi-quantification showed a high sensitivity but a lower specificity for COVID-19; ii) Histogram analysis of opacities zone on CT images could timely quantification of the heterogeneity, and is a potential approach for initial prognostic prediction and risk stratification for treatment decisions in COVID-19 patients; iii) nomogram based on COX hazard model with index derived from histogram analysis will archived accuracy prediction of the prognosis for an individual COVID-19 patient.

Page $8 / 19$ 


\section{Abbreviations}

COVID-19: coronavirus disease 2019;GGO: ground-glass opacity;CLO: consolidation opacity ;LOS: length of hospital stays;OS: overall survival;OR: odds ratio;ROC: receiver operating characteristic;ROI: region of interest;RTPCR: reverse transcription polymerase chain reaction

\section{Declarations}

\section{Acknowledgments}

We sincerely thank Dr. Shunan Wang and Hao Wu who worked in Department of Radiology, Daping Hospital Army Medical University for their great assistance in experimental design and statistical analysis.

\section{Author contributions}

YB and ZB collected all the clinical data and contributed to the structure and writing of thediscussion part. YB analyzed and interpreted the patient data and were also major contributor inwriting the manuscript. YB drafted the article or revised it critically for important intellectual content. ZJ and G LC contributed in patient screening, data collection. Q HM contributed to the background and interpretation of the data. H WC final approval of the version to be submitted. all authors agree to ensure any questions related to the work are appropriately resolved.

\section{Funding}

This work was supported by the General Social Development Project of Shaanxi Science and Technology Department囚2020SF-043囚.

\section{Data availability}

The datasets used and/or analyzed during the current study are available from the corresponding author on reasonable request.

\section{Ethics approval and consent to participate}

All procedures in this study were approved by the General Hospital of Central Theater Command (identification code, [2020]030-1; approved 23 April, 2020). written consent was waived by the Ethics Committee of the General Hospital of Central Theater Command.

\section{Consent for publication}

Not applicable.

\section{Competing interests}

The authors declare that they have no competing interests.

\section{References}


1. Zhu, N. et al. A Novel Coronavirus from Patients with Pneumonia in China, 2019. N Engl J Med 382,72733(2020).

2. Coronavirus COVID-19 Global Cases by the Center for Systems Science and Engineering (CSSE) at Johns Hopkins University (JHU). Retrieved from Johns Hopkins CSSE.(2020)..

3. Simpson, S. et al. Radiological Society of North America Expert Consensus Statement on Reporting Chest CT Findings Related to COVID-19. Endorsed by the Society of Thoracic Radiology, the American College of Radiology, and RSNA. J Thorac Imaging. .DOIه10.1097/RTI.0000000000000524(2020).

4. Inui, S. et al. Chest CT Findings in Cases from the Cruise Ship "Diamond Princess" with Coronavirus Disease 2019 (COVID-19). Radiology: Cardiothoracic Imaging 2,e200110(2020).

5. Hansell DM, Bankier AA, MacMahon H, McLoud TC, Müller NL, and Remy J. Fleischner Society: glossary of terms for thoracic imaging. Radiology. 2008;246(3):697-722.

6. B OH, Gransar H, Callister T, Shaw LJ, Schulman-Marcus J, Stuijfzand WJ, et al. Development and Validation of a Simple-to-Use Nomogram for Predicting 5-, 10-, and 15-Year Survival in Asymptomatic Adults Undergoing Coronary Artery Calcium Scoring. JACC Cardiovasc Imaging. 2018;11(3):450-8.

7. Eng, J, \& Bluemke, D,A. Imaging Publications in the COVID-19 Pandemic: Applying New Research Results to Clinical Practice. Radiology,201724(2020).

8. Radiology ACo. ACR Recommendations for the use of Chest Radiography and Computed Tomography (CT) for Suspected COVID-19Infection;

https://www.acr.org/Advocacy-and-Economics/ACR-Position-Statements/Recommendations-for-ChestRadiography-and-CT-for-Suspected-COVID19-Infection (2020).

7. Yu, H. et al. Computed tomography and pathology evaluation of lung ground-glass opacity. Experimental and therapeutic medicine 16,5305-9(2018).

8. Li, H. et al. SARS-CoV-2 and viral sepsis: observations and hypotheses. Lancet 395,1517-20(2020).

9. Xu, Z. et al. Pathological findings of COVID-19 associated with acute respiratory distress syndrome. Lancet Respir Med 8,420-2(2020).

10. Yaqian, M., Lin, W., Wen, J., a \& Chen, G. Clinical and pathological characteristics of 2019 novel coronavirus disease (COVID-19): a systematic review. medRxiv.2020.02.20.20025601(2020).

11. Park, J., Jung, J., Yoon, S,H., Goo, J,M., Hong, H., \& Yoon, J,H. Inspiratory Lung Expansion in Patients with Interstitial Lung Disease: CT Histogram Analyses. Sci Rep-Uk 8,15265(2018);.

12. Fu L, et al. Clinical characteristics of coronavirus disease 2019 (COVID-19) in China: A systematic review and meta-analysis. J Infect 80,656-65(2020).

13. Mao, R. et al. Manifestations and prognosis of gastrointestinal and liver involvement in patients with COVID19: a systematic review and meta-analysis. Lancet Gastroenterol Hepatol 5,667-678(2020).

14. Balachandran, VP, Gonen, M, Smith, JJ, \& DeMatteo, RP. Nomograms in oncology: more than meets the eye. Lancet Oncol 16,e173-e80(2015).

15. Zaki, AM, van., Boheemen, S., Bestebroer, TM., Osterhaus, AD., \& Fouchier, RA. Isolation of a novel coronavirus from a man with pneumonia in Saudi Arabia. N Engl J Med 367,1814-20(2012).

16. Li, Q. et al. Early Transmission Dynamics in Wuhan, China, of Novel Coronavirus-Infected Pneumonia. N Engl J Med 382,1199-207(2020). 
17. Huang, C. et al. Clinical features of patients infected with 2019 novel coronavirus in Wuhan, China. Lancet 395,497-506(2020).

18. Jin, J,M. et al. Gender Differences in Patients With COVID-19: Focus on Severity and Mortality. Frontiers in Public Health. https://doi.org/10.3389/fpubh.2020.00152(2020).

19. Choi, W,I., Rho, B,H., \& Lee, M,Y. Male predominance of pneumonia and hospitalization in pandemic influenza A (H1N1) 2009 infection. BMC Research Notes 4,351(2011).

20. Bernheim, A. et al. Chest CT Findings in Coronavirus Disease-19 (COVID-19): Relationship to Duration of Infection. Radiology 295,200463(2020).

21. Fang, L., Karakiulakis, G., \& Roth, M. Are patients with hypertension and diabetes mellitus at increased risk for COVID-19 infection? The Lancet Respiratory Medicine 8,e21(2020).

\section{Tables}

Table.1 Patients demographics, symptoms and clinical outcome. 


\begin{tabular}{|ll|}
\hline Parameters & Datum \\
\hline Age(y) & $67.00 \pm 18.89$ (rang from 31 to 97) \\
\hline Male to female ratio & $38: 19$ \\
\hline Initial symptoms & \\
\hline Fever & $48(84.2 \%)$ \\
\hline \multicolumn{1}{|c|}{ Hypodynamia } & $21(36.8 \%)$ \\
\hline Cough & $33(57.9 \%)$ \\
\hline \multicolumn{1}{|c|}{ Chest tightness } & $15(26.3 \%)$ \\
\hline Sore throat & $5(8.8 \%)$ \\
\hline Chills & $9(15.8 \%)$ \\
\hline Diarrhea & $7(12.3)$ \\
\hline Anorexia & $5(8.8 \%)$ \\
\hline Headache & $5(8.8 \%)$ \\
\hline Muscle pain & $13(22.8 \%)$ \\
\hline Clinical classification & $31.67 \pm 11.87$ \\
\hline Severe & $35(63.2 \%)$ \\
\hline Mild/Moderate & $21(36.8 \%)$ \\
\hline Clinical outcome & \\
\hline Discharged & \\
\hline Length of hospital stay (LOS) & $32(73.7 \%)$ \\
\hline Died & \\
\hline Overall Survival (OS) & \\
\hline
\end{tabular}

Table. 2 Frequency of lung abnormalities on CT and results of semi-quantitative score. 


\begin{tabular}{|c|c|c|c|c|c|}
\hline Parameters & $\begin{array}{l}\text { Total case } \\
\square n=57 \square\end{array}$ & Survival $\square n=42 \square$ & DiedIn=15! & Severe $\square n=36 \square$ & Mild\&moderate $\| n=21 \square$ \\
\hline \multicolumn{6}{|l|}{ Opacity frequency } \\
\hline Pure GGO & $13(22.8 \%)$ & $10(23.8 \%)$ & $3(20 \%)$ & $9(25.0 \%)$ & $4(19.0 \%)$ \\
\hline Pure consolidation & $8(14.0 \%)$ & $6(14.3 \%)$ & $2(13.3 \%)$ & $4(11.1 \%)$ & $4(19.0 \%)$ \\
\hline GGO+consolidation & $36(63.2 \%)$ & $26(61.9 \%)$ & $10(66.7 \%)$ & $23(63.9 \%)$ & $13(61.9 \%)$ \\
\hline \multicolumn{6}{|l|}{$\begin{array}{l}\text { Opacity } \\
\text { predominance }\end{array}$} \\
\hline GGO>consolidation & $33(57.9 \%)$ & $26(61.9 \%)$ & $7(46.7 \%)$ & $19(52.8 \%)$ & $14(66.6 \%)$ \\
\hline $\begin{array}{l}\text { Consolidation > } \\
\text { GGO }\end{array}$ & $24(42.1 \%)$ & $16(38.0 \%)$ & $8(53.3 \%)$ & $17(47.2 \%)$ & $7(33.3 \%)$ \\
\hline \multicolumn{6}{|l|}{$\begin{array}{l}\text { Frequency of lobes } \\
\text { affected }\end{array}$} \\
\hline Right upper lobe & $43(75.4 \%)$ & $29(69.0 \%)$ & 14(93.3\%) & $30(83.3 \%)$ & $13(61.9 \%)$ \\
\hline Right middle lobe & $42(73.7 \%)$ & $29(69.0 \%)$ & $13(86.7 \%)$ & $30(83.3 \%)$ & $12(57.1 \%)$ \\
\hline Right lower lobe & $53(93.0 \%)$ & $39(92.9 \%)$ & $14(24.6 \%)$ & $35(97.2 \%)$ & $18(85.7 \%)$ \\
\hline Left upper lobe & $43(75.4 \%)$ & $28(66.7 \%)$ & $15(100 \%)$ & $32(88.9 \%)$ & $11(52.4 \%)$ \\
\hline Left lower lobe & $50(87.7 \%)$ & $35(83.3 \%)$ & $15(100 \%)$ & $36(100 \%)$ & $14(66.6 \%)$ \\
\hline Total CT score & $6.14 \pm 3.9$ & $4.26 \pm 2.68$ & $7.41 \pm 4.18^{\star}$ & $7.44 \pm 4.01^{\#}$ & $3.90 \pm 2.44$ \\
\hline$p$-value & & 0.003 & & 0.000 & \\
\hline
\end{tabular}

\section{Figures}




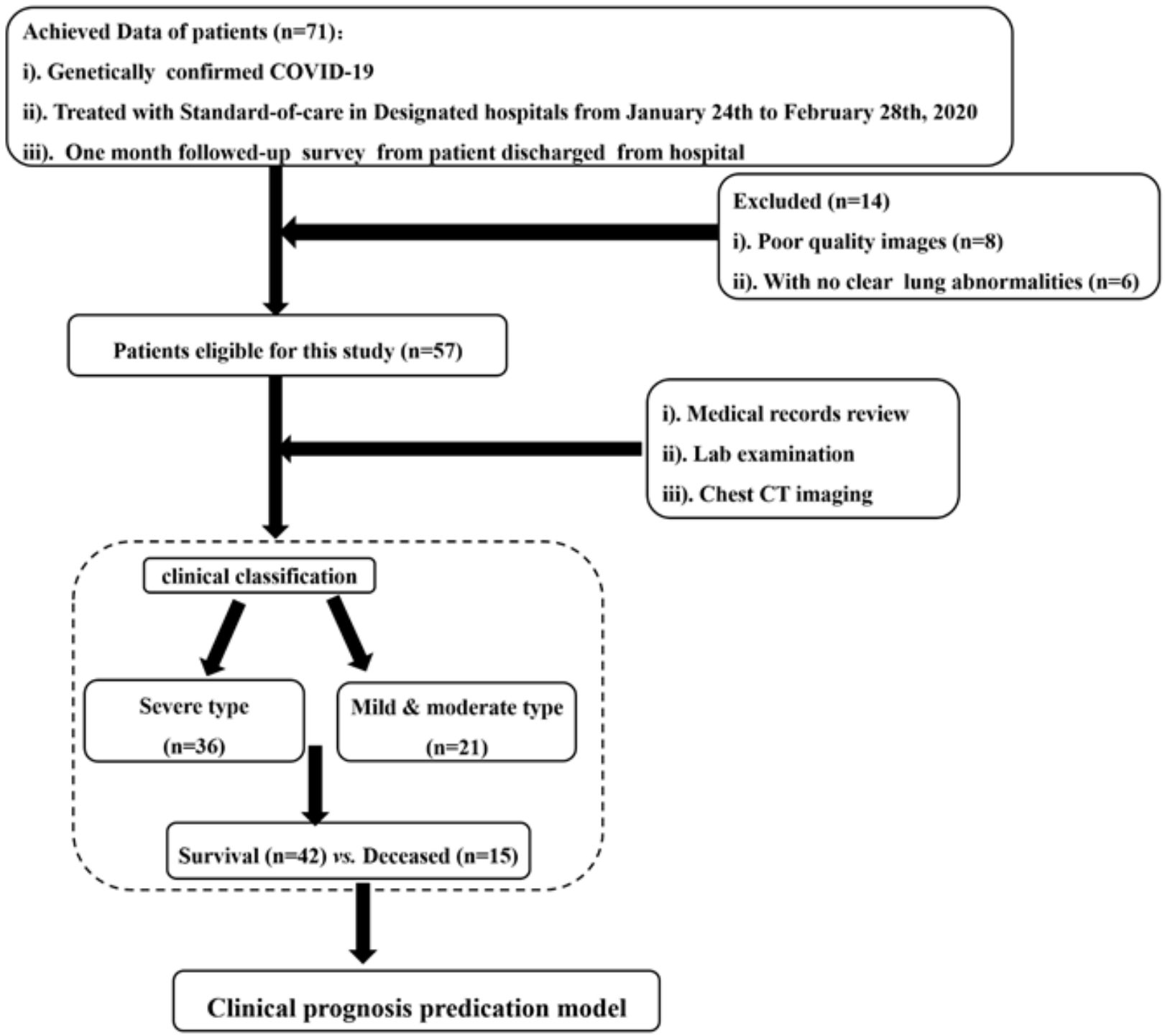

Figure 1

The retrospective study design and workflow diagram. 
ROC analysis for CT Semi-quantification in COVID-19 patients diagnosis
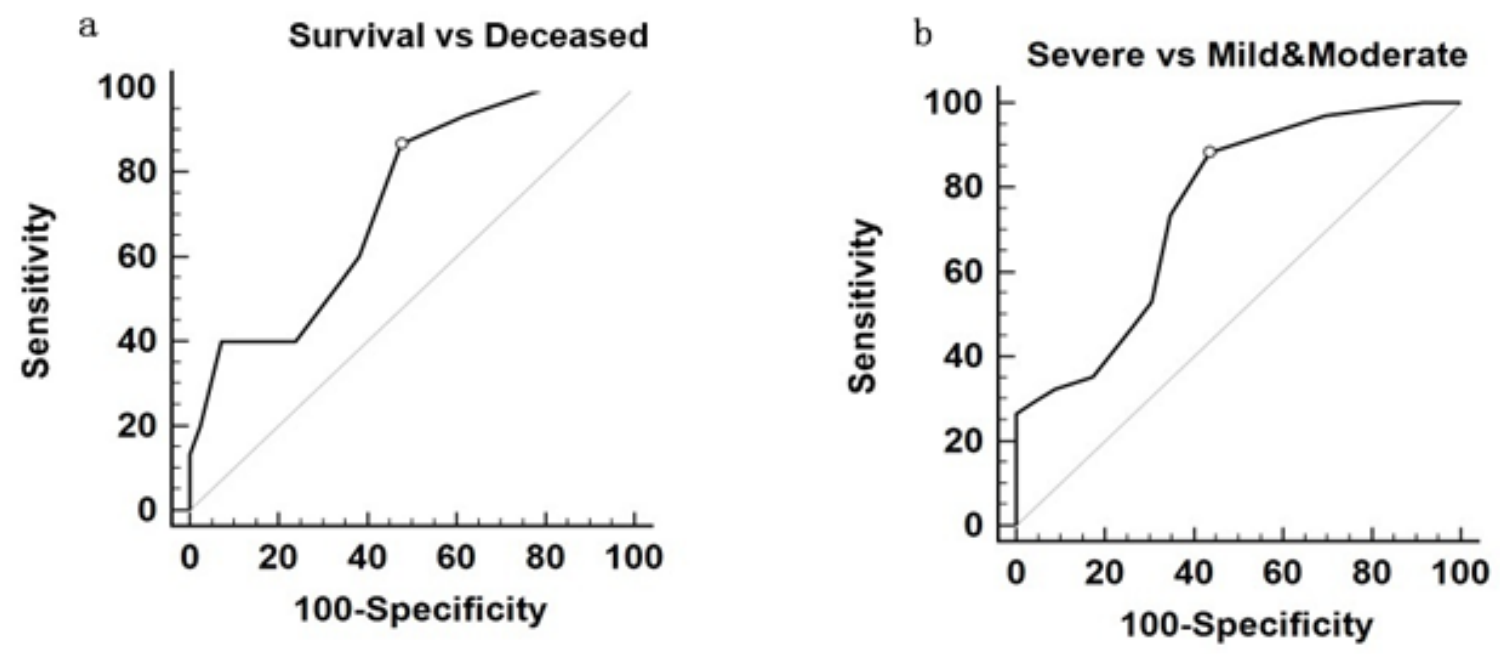

Figure 2

(a) ROC curves of discrimination of died population from their survival counterparts. (b) severe type in COVID-19 patients from mild/moderate type. 

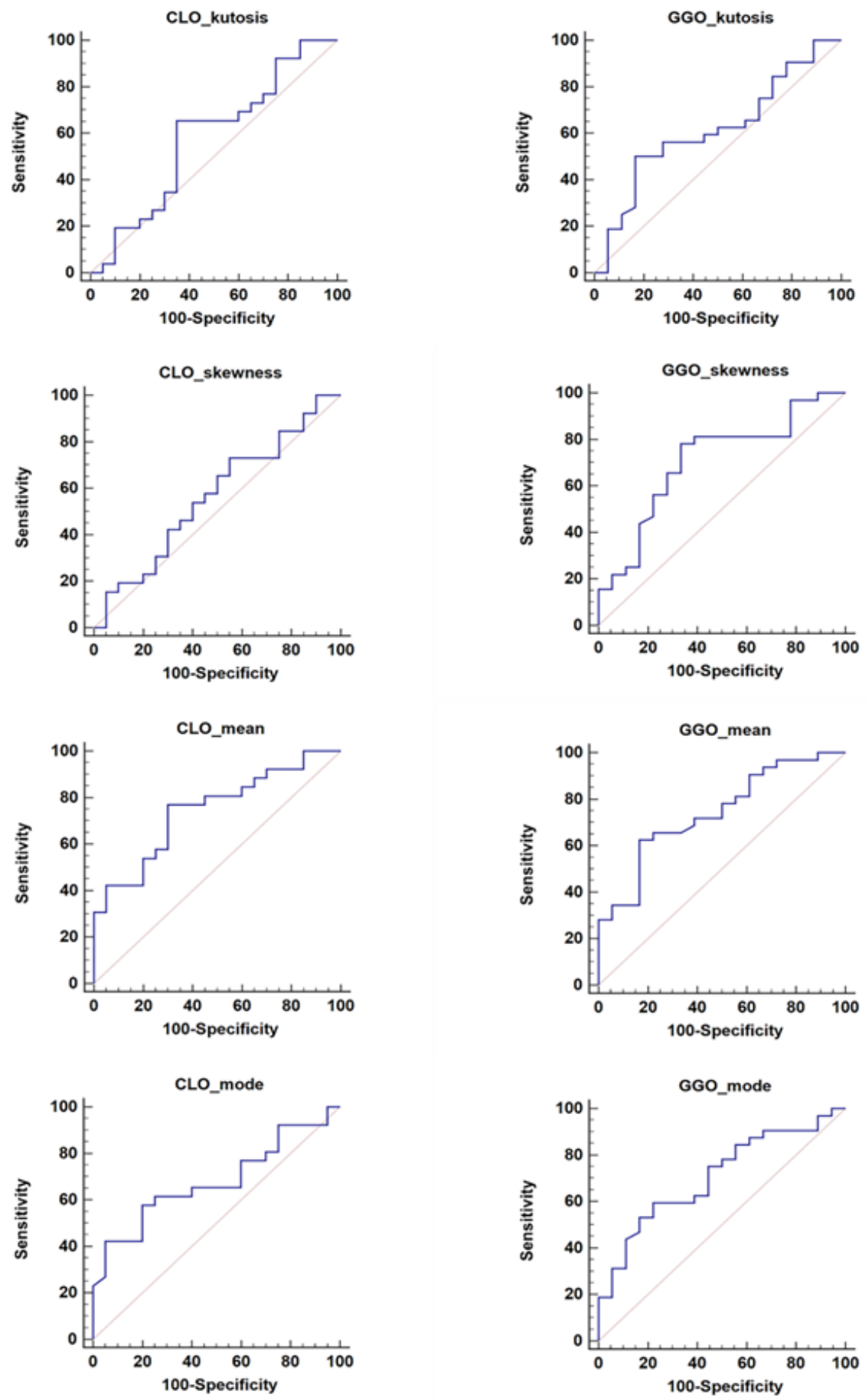

\section{Figure 3}

ROC curves of parameters derived from histogram analysis for discriminating severe type in COVID-19 patients. GGO, ground-glass opacity; CLO, consolidation opacity. 


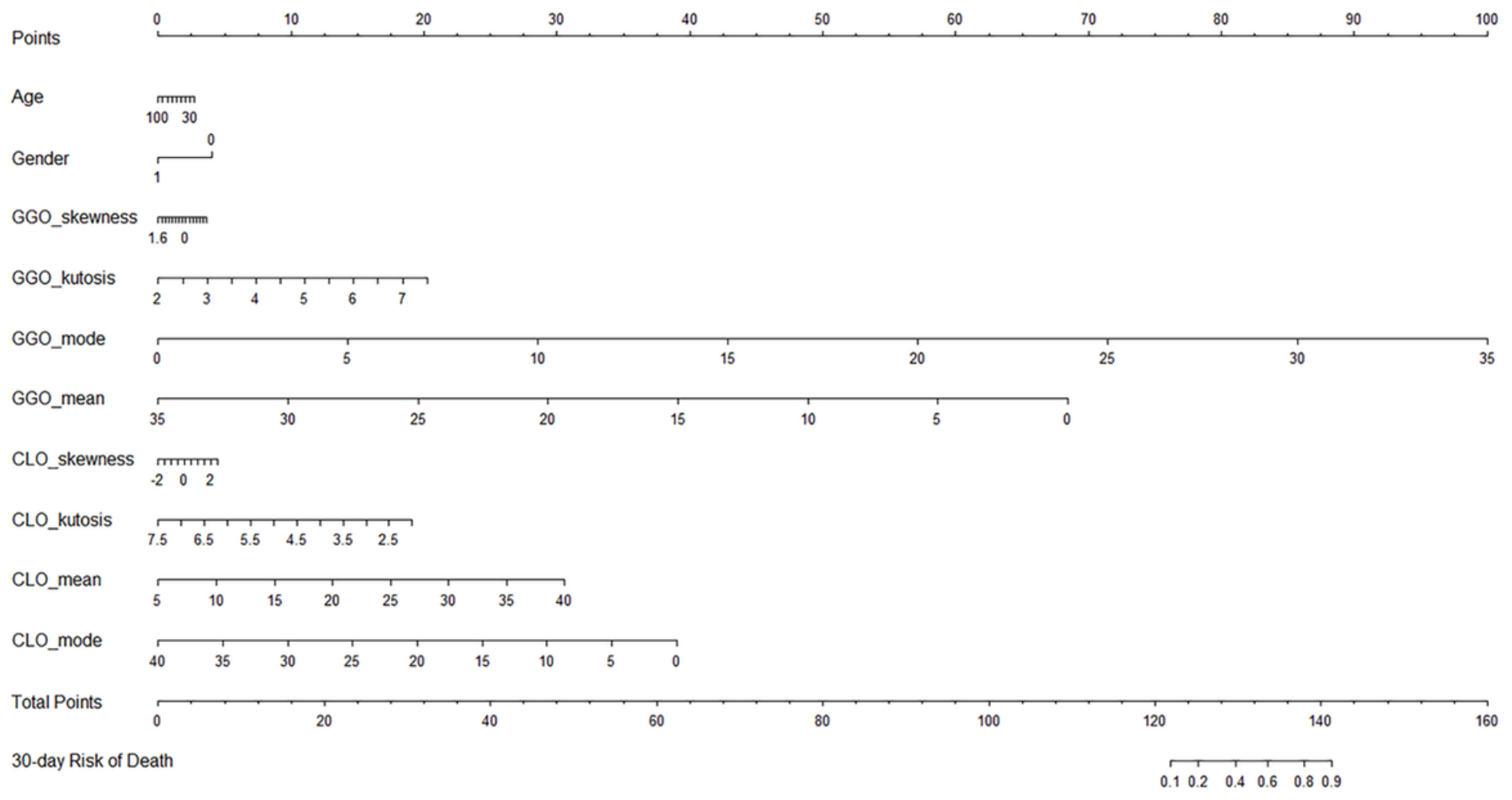

\section{Figure 4}

Nomograms convey the results of Cox proportional hazards regression model estimating 30-day risk of death for individual COVID-19 patients. 


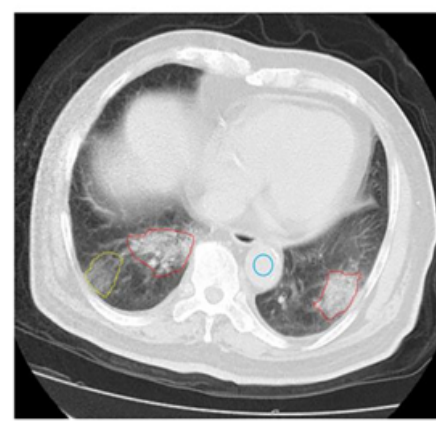

GGO

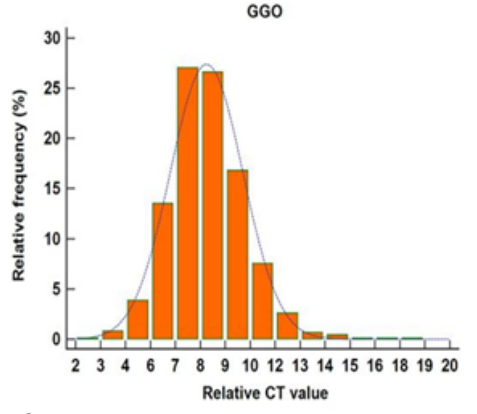

b
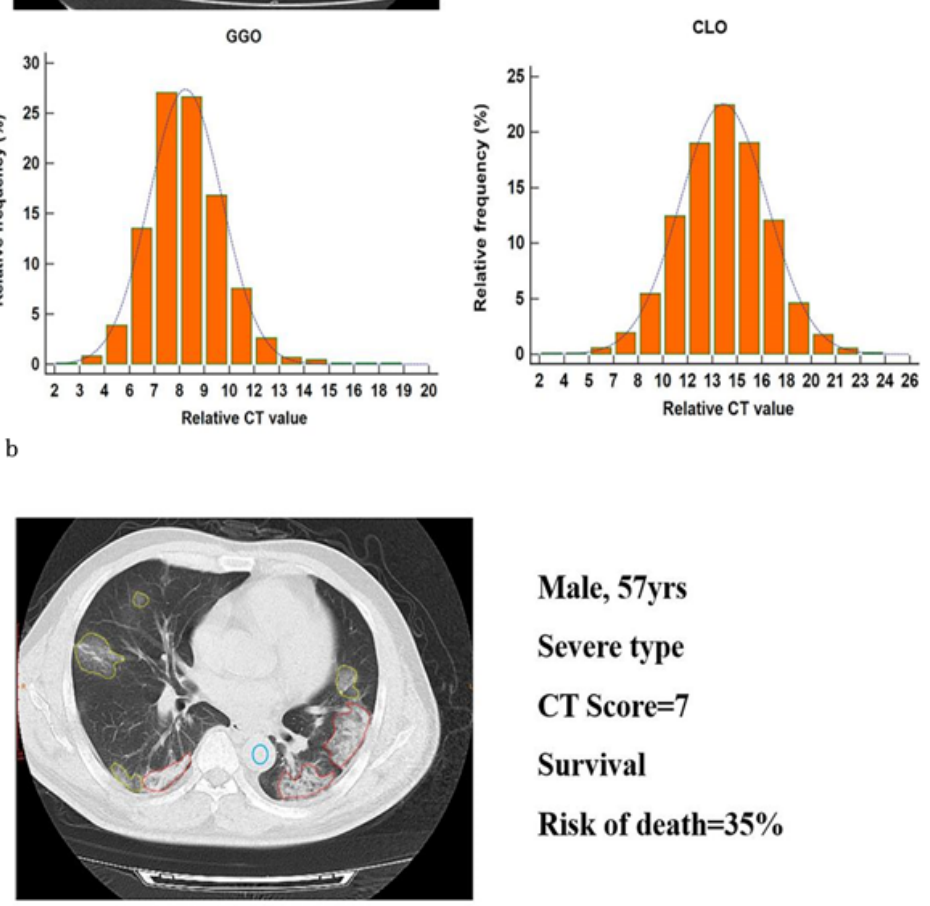

Female, 60 yrs

Severe type

CT Score $=7$

Deceased

Risk of death $=\mathbf{7 9} \%$

Male, $57 \mathrm{yrs}$

Severe type

CT Score $=7$

Survival

Risk of death=35\%
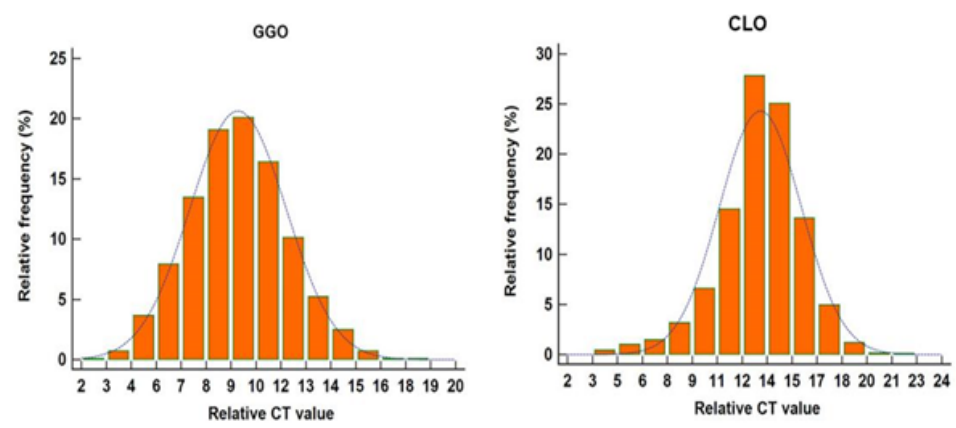

\section{Figure 5}

Example of individual prediction 30-day risk of death probability by nomogram in two patients with same CT score but different outcome.(a) a 60 years old female patient of died cohort with a total of histogram parameters $\approx 137$ points, which corresponds to a 30 -day risk of death probability of $79 \%$. (b) a 57 year old male patient of survival cohort with a total of histogram parameters $\approx 126$ points, which corresponds to a 30-day risk of death probability of $35 \%$. Red d otted circle, ROI of consolidation opacity; Yellow dotted circle, ROI of ground-glass opacity; Blue circle, ROI of thorax artery. 


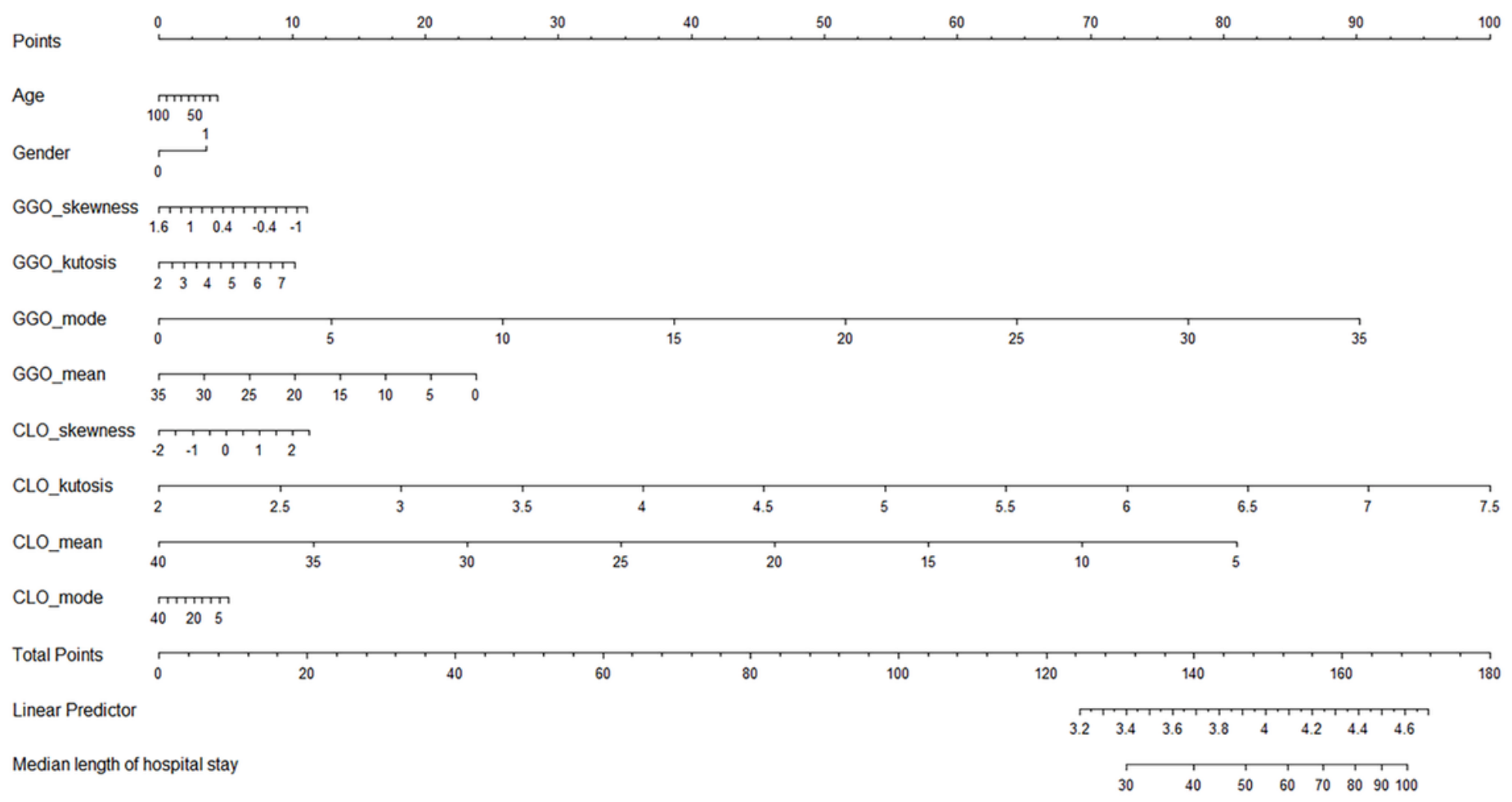

Figure 6

Nomograms convey the results of Cox proportional hazards regression model estimating length of hospital stays (LOS) for individual COVID-19 patients of survival population. The prolonged LOS (>30 days) was defined as the end-event.

\section{Supplementary Files}

This is a list of supplementary files associated with this preprint. Click to download.

- Additionalfile1.pdf 